\title{
PANGÉNESIS Y VITALISMO CIENTÍFICO
}

\author{
Ricardo Noguera Solano \\ Departamento de Biología Evolutiva, Facultad de Ciencias, UNAM, 04510 México, D.F. México
}

Rosaura Ruiz Gutiérrez

Secretaria de Desarrollo Institucional, UNAM, 04510 México, D.F. México

\begin{abstract}
RESUMEN
La hipótesis provisional de la pangénesis se ha interpretado como una explicación materialista y mecanicista de la herencia, seguramente porque Darwin mismo consideraba que las gémulas eran partículas corporales; sin embargo, analizando los principios en los que descansan las conjeturas de la pangénesis podemos ver cómo están entretejidas con nociones del vitalismo científico, principalmente el principio vitalista del nisus formativus de Blumenbach.
\end{abstract}

PALABRAS CLAVE: Pangénesis, vitalismo, Darwin, Blumembach.

\section{SUMMARY}

The provisional hipothesis of the pangenesis has been interpreted as a materialist and mechanistic explanation of heritance, surely because Darwin himself considered that the gemmules were corporal particles; nevertheless, analyzing the principles in which these suppositions on pangenesis rest we can appreciate how they are interlocked with notions of scientific vitalism, mainly Blumenbach's vitalist principle nisus formativus.

KEY WORDS: Pangenesis, vitalism, Darwin, Blumembach.

\section{INTRODUCCIÓN}

La hipótesis provisional de la pangénesis fue publicada en $1868^{1}$, Charles Darwin la propuso como una ley incluyente o general que determinara los principios de la generación, entendida en un sentido general como reproducción asexual y sexual, regeneración, hibridismo, desarrollo, atavismo y herencia.

1 DARwIn, Ch. (1868), The Variation of Animals and Plants Under Domestication, Vol. 2, pp. 349-399. 
Darwin imaginó a partir de ideas de fisiólogos del siglo XIX y de viejos supuestos de la generación que las unidades del cuerpo eran autónomas y propuso que cada una de ellas producía gérmenes o gémulas ${ }^{2}$ las cuales viajaban por los canales intracelulares y eran finalmente colectadas en los elementos sexuales, de esta manera: óvulos, espermatozoides, polen, huevos, semillas y brotes estaban constituidos por una gran cantidad de dichas partículas ${ }^{3}$.

Darwin pensaba que, así como una célula de una planta tiene la capacidad de producir un individuo en su totalidad ${ }^{4}$, cada uno de los elementos anteriores podía producir un organismo completo debido a las gémulas contenidas en ellos, cuando estos gránulos diminutos eran suministrados con sustancias nutricionales se multiplicaban y se desarrollaban en unidades como aquellas de las cuales habían derivado ${ }^{5}$, garantizando así la generación de las partes del cuerpo de donde las gémulas procedían.

Para Darwin, las gémulas eran partículas que daban continuidad a la vida, eran las responsables de la variación y eran las unidades últimas donde se generaban los cambios. Basado en esas suposiciones podía explicar entre otras cosas los problemas de la variación: causas, transmisión y manifestación de los caracteres. Las gémulas también tenían la capacidad de mantenerse activas generación tras generación o permanecer en estado de latencia, con ello explicaba la reaparición de caracteres ancestrales.

La variación o las ligeras diferencias entre individuos - explicaba Darwin- ocurren por diversas causas. En un primer caso, por la carencia o exceso de gémulas, y por la reiniciación del desarrollo de las que permanecen en estado de latencia, en estos procesos las gémulas no sufrían ningún cambio constitutivo. En otro conjunto de causas, la variación era resultado de la acción directa de las circunstancias que actuaba sobre el organismo; por el uso o desuso de las partes; por cambios innatos, imposible de relacionar con alguna causa concreta, y ocasionalmente por la hibridación de las gémulas, en estos segundos casos, las unidades modificadas segregaban gémulas modificadas, esto implicaba en el fondo cambios constitutivos de las gémulas y como consecuencia cambios en las células generadas por ellas.

2 Un término que Darwin heredó de Robert Grant uno de los profesores que más influyeron en sus concepciones como lo ha señalado Philip R. SLOAN, (1985) «Darwin's Invertebrate Program, 1826-1836 Preconditions for Transformism», pp. 71-120.

3 DARWIN (1868), Vol. 2, p. 357-358.

4 Ibidem, Vol. 2, p. 437.

5 Ibidem, Vol. 2, p. 370. 
Históricamente esta idea de la pangénesis ha sido vista como una teoría equivocada de la herencia ${ }^{6}$. Para nosotros, es un conjunto de principios generales, o como Darwin mismo pensaba, la pangénesis es una doctrina ${ }^{7}$ que aplicó a una problemática particular relacionada con la transmisión de la constancia y la variación de los caracteres, cuestiones vinculadas con el recién consolidado problema de la herencia, esto implica que Darwin comprendió la problemática de la herencia pero trató de explicarlas con un sistema de creencias equivocado ${ }^{8}$ articulado con los viejos problemas de la generación y el desarrollo.

Darwin estaba formado conceptualmente en el viejo programa de la generación por lo que no pudo evitar retomar ideas científicas relacionadas entre este programa y el vitalismo del siglo XVIII. El vitalismo al que nos referimos es la postura filosófica-científica, impulsada por Paul Joseph Barthez, quien postuló un «principio vital», de naturaleza desconocida, distinto de la mente y dotado de movimientos y sensibilidad, como la «causa de los fenómenos de la vida en el cuerpo humano». Este principio que fue retomado por muchos autores, entre ellos Darwin, fue en términos de Georges Canguilhem, la traducción «de una exigencia permanente de la vida en lo viviente»" ${ }^{9}$, fue también una postura contraria al mecanicismo como método científico y como filosofía reducida a la idea cartesiana del animal-máquina y a la reducción de los fenómenos orgánicos como resultado exclusivo de las fuerzas físico-

6 Por ejemplo, RAdL, E. (1930), The History of Biological Theories; SINGER, Ch. (1947), Historia de la Biologia; MAYR, E. (1982), The Growth of Biological Thought: Diversity; OlBy, R. (1985), Origins of Mendelism; y Bowler, P. (1989), The Mendelian Revolution. Estudios recientes la han considerado como una teoría del desarrollo y no de la herencia, por ejemplo, Hodge, M. J. S. (1985), «Darwin as a Lifelong Generation Theorist», en The Darwinian Heritage, pp. 207 - 244; y WINTHER, R. (2000), «Darwin on Variation and Heredity» Journal of the History of Biology, 33: 425-455.

7 DARWIN (1868), p. 400. Esta doctrina, ha sido revisada históricamente por C. Zirkle (1946) y E. Lesky (1951). Zirkle encuentra en los planteamientos de Darwin una continuidad con el pasado en cuanto a la concepción pangenética antigua: la idea de que un nuevo ser se origina a partir de pequeñas partes representativas de todo el cuerpo del que procede. Por su parte, Lesky analiza parte de estas ideas antiguas y considera que hay una gran similitud conceptual entre las ideas de Demócrito y Darwin.

8 En Noguera S. R. y Ruiz G. R. (en prensa), «Darwin and Inheritance», analizamos los cambios conceptuales de las reflexiones de Darwin sobre la herencia, en particular las influencias de las ideas del médico francés Prosper Lucas en las conjeturas darwinistas. Vease también, LóPez Beltrán, Carlos (1992), Human Heredity 1750 - 1870; The Construction of a Domain.

9 Canguilhem, G. (1976), El Conocimiento de la Vida, p. 99. 
químicas. Esta postura vitalista defendida desde el terreno de la historia por Canguilhem (1976) como una postura filosófica y como una propuesta de autonomía de la biología, fue compartida por científicos que hicieron aportes fundamentales en distintos problemas de la biología, como la construcción de la teoría celular, la explicación del desarrollo embriológico, y la explicación del arco reflejo ${ }^{10}$. En el fondo ser vitalistas en este periodo, no significó de ninguna manera frenar la investigación científica, algo similar ocurrió con Darwin, que a pesar de haber recurrido a ideas vitalistas - como sostenemos en este trabajo - no impidió que llegara a comprender que la variación no sólo era una característica inherente a lo vivo, sino era también algo esencial para su evolución.

Entre los personajes participantes de este vitalismo científico se encuentran Johann Friedrich Blumenbach y James Paget ${ }^{11}$, autores cuyas ideas utilizará Darwin para respaldar sus argumentos. Para estos dos autores como para Darwin, el «principio vital» estaba distribuido en todas las partes de los seres. En Blumenbach es claro que este principio vital tiene una participación definitiva en todos aquellos aspectos de la vida que muestran (o parecen mostrar) alguna forma de programa o comportamiento dirigido a metas predeterminadas; en Darwin esta teleología puede ser discutida.

J. F. Blumenbach (1752-1840), fue un médico, antropólogo y naturalista alemán considerado como fundador de la antropología física. En 1775 utilizó el término «raza» para clasificar los grupos humanos, primero en cuatro grupos y posteriormente en cinco. Entre sus ideas fundamentales estaba la sugerencia de considerar a los seres humanos como parte de los objetos de la historia natural y veía en ellos «al más perfecto de todos los animales domésticos». La preocupación de Blumenbach sobre la naturaleza era una inquietud filosófica comprometida con la investigación de las causas de las cosas; en un esfuerzo por rechazar ideas preformacionistas llegó a elaborar la idea del nisus formativus publicada en 1779, ésta causó gran impresión entre sus contemporáneos y en algunos científicos posteriores, entre ellos Darwin. Con esta noción vitalista, Blumenbach ofreció argumentos a favor de la epigénesis y de ciertos planteamientos evolutivos finalistas.

10 Ibidem, p. 104-111.

11 James Paget (1814-1889), médico y cirujano inglés, publicó diferentes trabajos sobre patología y cirugía. Paget es otro de los fisiólogos que Darwin señala junto con Blumenbach, que insiste sobre la existencia de un mismo principio, presente en la regeneración de las hidras $\mathrm{y}$ en las cicatrizaciones de los organismos superiores; ese principio o poder es el nisus formativus, que Paget también heredó del médico alemán. 
Las ideas que exploramos fueron conocidas por Darwin en la misma época del nacimiento de la hipótesis de la pangénesis ${ }^{12}$, Darwin las conoció durante las lecturas que hiciera en $1839^{13}$, esas fueron: An Essay on Generation de Blumenbach ${ }^{14}$; An Essay on Animal Reproduction de Lazzaro Spallanzani ${ }^{15}$ y Zoonomia: or the Laws of Organic Life de Erasmus Darwin (1794).

\section{EL PRINCIPIO VITALISTA}

En el manuscrito sobre la pangénesis de $1865^{16}$ Darwin considera que los organismos están constituidos por una multitud de partes elementales independientes unas de otras, así como los elementos de reproducción: gérmenes y óvulos ${ }^{17}$; también suponía que todos estos elementos contenían una materia formativa $^{18}$ o protoplasma. En este mismo manuscrito señala cómo algunos problemas de la generación, por ejemplo, la regeneración de extremidades amputadas y la cicatrización de heridas entre otros problemas, estaban involucrados con lo que los viejos fisiólogos llamaban el nisus formativus ${ }^{19}$. Como indicamos anteriormente, el nisus formativus, es un término que Darwin retoma de Blumenbach, pero no sólo utiliza el vocablo, retoma también el concepto.

El punto principal de nuestra discusión consiste en señalar que la supuesta materia formativa tanto del escrito de 1865 sobre la pangénesis como de las

12 Sobre el origen de la pangénesis puede consultarse HodGe, M, J. S. (1985) y HodGe, M. J. S. (1989) «Generation and the Origin of the Species (1837-1937): A Historiographical Suggestion», BJHS, pp. 267-281.

13 De acuerdo a la cronología elaborada por VorzIMMER, P. J. (1977), The Darwin Reading Notebooks (1836-1860), p. 121.

14 La obra de Blumenbach que Darwin menciona, es la traducción al Inglés de Alexander Crichton, publicada en 1792.

15 Lazzaro Spallanzani (1729-1799), profesor de historia natural y sacerdote jesuita, motivado por las ideas preformacionista se dedicó a investigaciones de la generación, realizó experimentos para rebatir la hipótesis de J. T. Needham sobre la generación espontánea. La obra que Darwin señala haber leído es la traducción al inglés del trabajo de Spallanzani de 1769.

16 DARwin, Ch. (1865), «Manuscript of pangenesis», transcripción de OlBY, R. C. (1963), Charles Darwin's Manuscript of Pangenesis, pp. 250-263.

17 En la publicación de 1868 adicionará: brotes y semillas.

18 En el capítulo de la pangénesis utiliza la idea de materia formativa, la cual considera: «está dispersa a lo largo de todos los tejidos», esta materia formativa estaba constituida de partículas o gémulas producidas en cada unidad o célula. DARWIN (1875), The Variation of Animals and Plants Under Domestication, Vol. 2, p. 372.

19 DARWIN (1865), «Manuscript of Pangenesis», p. 256. 
versiones de 1868 y 1876 de su libro The Variation of Animals and Plants Under Domestication (en adelante The Variation) está relacionada con la idea del nisus formativus ${ }^{20}$ y de manera discreta con una idea vitalista, tal y como puede interpretarse del siguiente comentario:

«El protoplasma o materia formativa incluida dentro de los gérmenes y elementos masculinos y femeninos, está dotado de una fuerza vital, ésta y la generación seminal son causa del desarrollo de cada ser, así como de los siguientes atributos: la herencia, la reversión y la hibridación» ${ }^{21}$.

En esta reflexión hay una idea de autosuficiencia e independencia de la materia formativa respecto a los órganos reproductores masculinos y femeninos. Como lo expondrá con mayor detalle en las publicaciones de 1868 y 1875 , los elementos reproductores no generan protoplasma, la materia formativa difundida por todo el organismo es derivada de cada tejido o cada tipo de tejido, los elementos reproductores sólo la seleccionan y la acumulan en cantidades apropiadas y la dejan lista para una existencia independiente.

En el primer borrador de la pangénesis (1865) las gémulas, protoplasma y átomos eran sinónimos de la materia formativa y cualesquiera de esos elementos juntos en proporciones apropiadas eran los elementos constitutivos del germen verdadero 22 .

Algunos antecedentes del manejo de Darwin del nisus formativus están también en el manuscrito de la selección natural de 1856 cuyos primeros dos capítulos están dedicados al problema de la variación ${ }^{23}$. Los contenidos son muy parecidos a los que aparecen en los capítulos de The Variation. El apartado 35 del manuscrito de la selección natural dedicado a las leyes que regulan la variación contiene una discusión sobre el equilibrio orgánico y el nisus formativus.

Como adelantamos párrafos atrás, en 1839 Darwin tiene un notable interés por la materia formativa relacionada con los problemas de la generación, por ello lee a Blumenbach, y también a Spallanzani y conservará de estos autores las ideas que utilizará muchos años después a favor de sus argumentos de la pangénesis.

20 Ibidem, p. 256, y en DARWIN (1868), Vol. 2, p. 293.

21 DARWIN (1865), p. 258.

22 Ibidem, p. 259.

23 Este manuscrito fue publicado por: STAUFFER, R. C. (1987), Charles Darwin, Natural Selection, Being the Second Part of his Big Species Book Written from 1856 to 1858. 
Lo importante para nuestra argumentación es que no solo mantuvo ideas similares de la pangénesis en sus distintas reflexiones escritas, sino además, en la segunda edición de The Variation (1875) insertó un hecho más para fortalecer sus argumentos sobre la importancia de los problemas de la generación, la materia formativa, el nisus formativus y la pangénesis, este hecho adicional fue la regeneración de partes amputadas respaldando sus afirmaciones con los experimentos de Spallanzani y Charles Bonnet ${ }^{24}$, del primero cita sus observaciones de regeneración en salamandras ${ }^{25}$, experimentos que por algún motivo no consideró necesario incluirlos en la primera edición.

En estos párrafos adicionados señalará que el poder de regeneración es mucho más grande en animales jóvenes, sobre todo en las primeras etapas de su desarrollo, y que los organismos inferiores en la escala, son capaces, como regla general de reproducir partes perdidas más fácilmente que aquellos que tienen una organización superior. También señalará que aquellos organismos que son divididos en dos o incluso cortados en pedazos son capaces de reproducir un nuevo ser de manera completa - esto es posible dice Darwin- porque el poder de la regeneración debe estar difuso a través de todo el cuerpo ${ }^{26}$.

Ese poder de regeneración no era otra cosa que el nisus formativus de Blumenbach. Para el médico alemán, el nisus formativus era la fuente de toda generación y reproducción en cada reino organizado, este poder vital poseía la capacidad de organización, moción y sensación ${ }^{27}$ con el cual podía explicarse fenómenos orgánicos como el crecimiento y la re-generación.

En The Variation (1868), Darwin señala estas ideas en el apartado de «Las leyes de la variación ${ }^{28}$ y en la edición de 1875 , mantiene el mismo discurso en los mismos capítulos. Un dato que en apariencia pudiera restar fuerza a nuestra argumentación radica en el hecho de que en el capítulo XXVII dedicado a la pangénesis no aparece el término nisus formativus, palabra que sí aparece en el manuscrito de 1865; pero interpretamos que el nisus formativus de los viejos fisiólogos fue intercambiado por la idea de materia formativa. Un cambio muy importante por las implicaciones que podría tener la idea de «materia», esto ha motivado la creencia de que la hipótesis provisional de la pangénesis fue una explicación científica completamente materialista. Esta

24 Bonnet, Ch. (1781), Euvres d'Hist. Nat. tomo v., parte I, cuarta edición. pp. 343, 350. Citado por DARWIN (1875), Vol. 2, p 357.

25 DARWIN, Ch. (1875), Vol. 2, pp. 283, 357, 358.

26 DARWIN (1875), Vol. 2, p. 358.

27 Blumembach, J. F. (1828), The Elements of Physiology, p. 29.

28 DARWIN (1868), pp. 293 y 294. 
idea de materia formativa, ya era utilizada por Darwin en el manuscrito de $1865^{29}$. Nuestro argumento se refuerza si consideramos que en los capítulos sobre las leyes de la variación de ambas ediciones de The Variation (1868, 1875) el nisus formativus y la materia formativa es utilizada en el mismo sentido, como un principio involucrado en los problemas de la generación, entendida en todos los sentidos que Darwin le asigna.

Opinamos que Darwin retomó esta idea vitalista en sus reflexiones, quizá con una pequeña diferencia. Para Blumenbach el nisus formativus era:

«Un impulso distinto por completo de todas las fuerzas formativas meramente mecánicas [...] capaz de modificar los distintos tipos de material seminal organizable de diversos modos, aunque dirigidos a un objetivo, y combinarlos en formas determinadas ${ }^{30}$.

Darwin no expresa la idea direccional de Blumenbach y no deja claro si este principio es distinto de las fuerzas meramente mecánicas o físicoquímicas, a pesar que tuvo la oportunidad de aclararlo ante la critica de Federico Delpino (1869) quien señalaba que la pangénesis era una explicación materialista que rechazaba la intervención de un principio específico sui generis inherente a los procesos orgánicos, suponiendo que las designadas fuerzas vitales no eran sino transformaciones de las llamadas fuerzas físicoquímicas $^{31}$. Darwin parece mantenerse a distancia de la discusión, señalando en ambas ediciones de The Variation, las posturas encontradas sobre la formación de células; por un lado, las ideas de los partidarios de la doctrina celular, la idea de que las células se originaban gracias a un poder inherente que las células poseían ${ }^{32}$, o que las células se generaban a partir de otras preexistentes; también señala la postura opuesta: que las células y tejidos de todos los tipos podían ser formados a partir de linfa plástica o blastema de manera independiente de células pre-existentes.

Darwin mantuvo una postura un tanto ambigua ante esta discordancia que reflejaba de alguna manera el debate entre vitalistas y mecanicistas, para no comprometerse, escribió: «como no me ocupo especialmente de la histología, podría ser presuntuoso expresar alguna opinión sobre estas doctrinas opuestas $\rangle^{33}$.

29 Ver citas 16 y 17.

30 Blumembach, J. F. (1830), Handbuch der Naturgeschichte, pp. 15-17. Citado en RoBERT, J. R. (1992), The Meaning of Evolution, p. 38.

31 Delpino, F. (1869), On the Darwinian Theory of Pangenesis, p. 407.

32 DARWIN (1868), Vol. 2, p. 370.

33 Idem. 
Este acto de modestia no es tal, ya que Darwin contaba con su propia versión respecto a la explicación de la generación de nuevas células o unidades de vida, ya fuera en cualquiera de los tipos de reproducción seminal y asexual, así como en los procesos de regeneración e hibridación. En su explicación Darwin recurrió a un principio según suponía, debía ser fundamental en estos fenómenos, la admisión de un principio generativo, coordinante y organizativo (en este caso el nisus formativus) que pasaba mediante cualquiera de los diferentes tipos de generación en los diversos linajes, desde sus más lejanos ancestros.

Al inicio de los capítulos de Las leyes de la variación, en la edición de 1868, Darwin considera necesario discutir el poder de coordinación y reparación de la organización, la cual dice:

«Es común en un mayor o menor grado a todos los seres orgánicos, un poder que formalmente ha sido designado por los fisiólogos como nisus formativus. Blumenbach y otros han insistido que el principio el cual permite a la Hidra que se desarrolle a sí misma en un animal perfecto es el mismo poder que permite la cicatrización en animales superiores» ${ }^{34}$.

Darwin desarrolla un conjunto de ideas similares en la primera parte del capítulo de la pangénesis y será precisamente en relación con el mismo conjunto de problemas relacionados con la generación: creación de un nuevo ser, por cualquiera de los tipos de reproducción, regeneración, hibridación y cicatrización; para él no hay diferencias entre el poder que interviene en todos esos casos. Este conjunto de problemas es el mismo que presenta en el capítulo de las leyes de la variación, en ambos casos señala que hay un poder coordinante, un nisus formativus o una materia formativa, incluso también lo designa como «poderes del desarrollo» como puede verse en las siguientes líneas que Darwin utiliza de Paget para reafirmar sus convicciones:

«Paget admite, que los poderes del desarrollo del embrión son idénticos a los involucrados en la reparación de lesiones [... Los poderes son los mismos tanto en el logro de la perfección como en su recuperación cuando ésta se pierde» ${ }^{35}$.

Ese supuesto poder está involucrado en las distintas formas de gemación, generación fisípara, en reparación de lesiones, en el mantenimiento de cada parte en su estado correcto, en el crecimiento o desarrollo progresivo de toda la estructura del embrión; debido a ello, todas estas formas de generación, dice Darwin: «son

34 Ibidem, Vol. 2, p. 293.

35 Ibidem, Vol. 2, p. 359. 
resultado esencialmente de uno y el mismo gran poder» ${ }^{36}$. Esta idea permaneció sin cambios sustanciales en la segunda edición de The variation ${ }^{37}$.

¿Cómo entiende Darwin el papel de este gran poder? Un poder que no siempre actúa en forma perfecta en el proceso de la regeneración. Estas ideas son similares en los capítulos de las leyes de la variación y en el de la pangénesis. En el primero de estos capítulos su idea del «gran poder» es claramente la idea del nisus formativus, y es explicado sin ambigüedades a través de la autoridad de Blumenbach:

«La actividad del nisus formativus está en proporción inversa a la edad del cuerpo organizado. Este poder también es mayor en animales de peldaños inferiores en la escala de la organización, y los animales inferiores en la escala corresponden con los embriones de animales superiores que se siguen en la misma clase $»^{38}$.

Darwin considera que bajo el encabezado de fenómenos relacionados sobre el nisus formativus, no sólo deben considerarse las viejas estructuras que son capaces de reproducirse, sino también las estructuras que son formadas de nuevo, por ejemplo, la inflamación, en donde las falsas membranas, dice, son suministradas con vasos sanguíneos, vasos linfáticos y nervios, un segundo ejemplo de estas estructuras nuevas es la membrana con la que se nutre el feto que está ricamente suministrada por vasos sanguíneos ${ }^{39}$. Inmediatamente después de señalar ambos ejemplos y el nisus formativus Darwin adiciona una reflexión contrapuesta que le permite señalar la discusión que representan las investigaciones del desarrollo de la teoría celular, el párrafo que a continuación citamos se mantiene idéntico en la segunda edición:

«pero, muchos fisiólogos del continente tienen ahora la creencia en una linfa plástica o blastema ${ }^{40}$, y Virchow ${ }^{41}$ mantiene que cada estructura nueva o vieja es

36 De la obra Lectures on Surgical Pathology, Vol. 1, London; Longman, 1853, citado en DARWIN (1868), p. 359.

37 DARWIN (1875), p. 352.

38 DARWIN (1868), Vol. 2, p. 294. En los márgenes del libro de Paget Lectures on Surgical Pathology, 1853, Darwin anotó una idea similar: «El poder de reparación es inversa a la cantidad de poder, ya consumido en el desarrollo de un individuo». Di GREGORIO, M. (1990), Charles Darwin's Marginalia, Vol, 1, p. 659.

39 DARWIN (1868), Vol. 2, p. 294.

40 Darwin se refiere al citoblastema, término acuñado por Theodor Schwann (18101882), para referirse a una matriz de estructura amorfa, granulada y generalmente de consistencia viscosa, a partir de la cual se formaban las nuevas células.

41 Rudolf L. Virchow (1821-1902). El modelo de formación de células estudiado por Robert Remak fue generalizado por Virchow en la segunda mitad del siglo XIX, para Virchow, 
formada por la proliferación de células preexistentes» ${ }^{42}$

Como adelantábamos en páginas anteriores, Darwin no comparte del todo los principios de la teoría celular, y en este párrafo refleja que a pesar de su escepticismo con respecto a esa teoría, no existe para él un vacío explicativo referente a los problemas de la generación, porque su idea del nisus formativus ocupa el lugar de dicha teoría. Es evidente que no le concede a la teoría celular el espacio adecuado, ni en la primera ni en la segunda edición porque señala que: «incluso el gran defensor de la doctrina: omnis cellula e cellula, Virchow, manifiesta que existen dificultades para mantener que cada átomo de tejido sea derivado de células, y éstas de células preexistentes, y éstas a partir del huevo, el cual se considera como una gran célula. A pesar de que en ambas ediciones señala que la teoría (celular) está aceptada como unidad general en el campo del estudio de las plantas, y está ampliamente aceptada en los animales ${ }^{43}$, y muy a pesar de que los defensores de la doctrina celular recurrían a un poder inherente a las células y no a un agente externo; ${ }^{44}$ Darwin acepta la teoría a medias, sólo está convencido de que la composición y organización de los organismos es resultado de miles de pequeñas unidades orgánicas. ${ }^{45}$ En su reflexión sólo hay afinidad con respecto a uno de los principios: las células son las unidades fundamentales de la organización, y hay un rechazo del segundo supuesto relacionado con la génesis de los organismos; toda célula deriva de una célula anterior ${ }^{46}$, en su lugar opta por hacer uso de un principio adicional, el nisus formativus. En su explicación una célula podía tener alguna continuidad con otra célula, pero no derivar propiamente de ella. Esta interpretación de Darwin, es distinta a la de los defensores de la teoría celular y a la de los partidarios que mantenían que las células podían formarse independientemente de células pre-existentes, a partir de ciertos cambios químicos. Sólo acepta que los cuerpos están constituidos de una multitud de «unidades orgánicas» cada una de las cuales posee sus propios atributos y es

el desarrollo embrionario era por divisiones celulares. A partir de una célula: el huevo formado por la madre. Virchow rechazó la interpretación del citoblastema primitivo de Schwann.

42 DARWIN (1868), Vol. 2, p. 295.

43 Ibidem, Vol. 2, p 370. En la segunda edición expresa la misma afirmación. Darwin (1876), Vol, 2, p. 377.

44 DARWIN (1868), Vol. 2, p. 370.

45 Basados en el análisis que Canguilhem hace de la construcción histórica de la teoría celular. CANGUILHEM (1976), p. 47-91.

46 Estos dos puntos fueron reunidos por Virchow, la primera era una suposición de Schwann. CANGuilhem (1976), p. 77. 
en cierta manera independiente de las otras, de ahí que considera que puede ser conveniente usar de manera indistinta los términos «células» o «unidades orgánicas» o simplemente «unidades» ${ }^{47}$. Darwin señala que «unidad orgánica» era un término del Dr. E. Montgomery, quien rechazaba que las células se generaran de células pre-existentes y creía que las células se originaban a través de ciertos cambios químicos ${ }^{48}$. En la segunda edición de The Variation, Darwin eliminó esta aclaración.

Para llenar el espacio que varios autores concedían a la teoría celular Darwin utilizó la idea del gran poder organizativo de sus gémulas y de la materia formativa, como señalábamos párrafos atrás, la materia formativa para Darwin en su manuscrito de la pangénesis estaba dotado con un poder vital; en sus discusiones sobre el nisus formativus Darwin elimina la idea de poder vital y solo conserva la idea de un gran poder: por otro lado, considerará en The Variation, tanto en la primera como en la segunda edición que la materia formativa está constituida por gémulas.

Era tan importante la idea de este poder organizativo y coordinante tanto para los problemas de la generación como para los problemas de la variación que Darwin expresa la siguiente consideración cuando inicia los capítulos sobre las leyes de la variación:

«Este tema - [el del nisus formativus] — ha sido citado aquí, porque podemos inferir que cuando alguna parte de un órgano es aumentada o disminuida continuamente por la variación y la selección, el poder coordinante de la organización, tiende continuamente a mantener a todas las partes en armonía» ${ }^{49}$.

Incluirá también una idea similar en el resumen de los mismos capítulos:

«Las modificaciones generadas por cualquier causa, serán reguladas hasta cierto punto por el poder coordinante o el supuesto nisus formativus, que es en realidad un remanente de la más simple forma de reproducción $»^{50}$.

En la segunda y última edición The Variation, Darwin considera que las gémulas no se desarrollan independientemente en células libres, sino que siempre se desarrollan en unión con «nascent cell» pre-existentes. ¿Significa esto que Darwin reconsideró de una manera diferente los principios de la teo-

47 DARWIN (1868), Vol. 2, p. 371.

48 Ibidem, Vol. 2, p. 370.

49 Ibidem, Vol. 2, p. 295. Los corchetes son nuestros.

50

DARWIN (1875), Vol. 2, p. 348. 
ría celular? Para 1875 la teoría celular gozaba ya de una buena aceptación, Darwin como lo señalamos también compartía uno de esos principios (todos los seres están formados por células), pero a pesar de que reelaboró en gran parte el capítulo sobre la pangénesis no hizo modificaciones en su discusión sobre el nisus formativus; ni dio visos de aceptar el segundo principio (toda célula proviene de otra célula). La introducción de la idea de «nascent cell» como elementos necesarios en la formación de nuevas células, no elimina la participación indispensable de las gémulas y en el fondo de la materia formativa.

A pesar de la persistencia de un discreto vitalismo, paradójicamente la pangénesis fue un avance en la explicación materialista o material del problema de la herencia; como lo reconocerá Hugo De Vries (1910), quien además dirá que esa hipótesis fue la inspiración ${ }^{51}$ que lo llevó a desarrollar su investigación sobre el problema.

El vitalismo de Darwin, como de algunos naturalistas, es un vitalismo diferente al vitalismo trascendental; cuyo postulado fundamental consiste en considerar a la vida como un fenómeno irreductible a dimensiones puramente físicas y/o químicas como creía Georges L. L. conde de Buffon ${ }^{52}$ y Caspar Friedrich Wolff ${ }^{5}$.

Para Darwin, como para Blumembach, el «principio vital» no es una idea trascendentalista a diferencia del animismo postulado por Georg Ernst Stahl en el siglo XVII en Alemania, y de donde se inspiraría este vitalismo biológico. La similitud consiste en que para ambos, el elemento inmaterial que postulan, el gran poder, como lo repite continuamente Darwin, representaba una

51 Estas ideas también influyeron en Francis GALTON (1971), «Experiments in Pangenesis», 19: 393-410, quien no compartió los puntos de vista de Darwin respecto a que las gémulas estuvieran en todo el cuerpo. Otro trabajo crítico fue el del botánico Italiano Federico DELPINO (1869), quien enfatizaba su comprobación en el terreno de la investigación experimental. Con el propósito de perfeccionar la hipótesis de Darwin Carl NAEGELI (1884), Mechanischphysiologische Theorie der Abstammungslehre, estudió el soporte material de la transmisión hereditaria en su teoría fisiológica - mecánica del evolucionismo en la que se conjetura la presencia de los factores hereditarios en la organización del óvulo. Otra hipótesis distinta sobre la transmisión hereditaria, inspirada tanto por el Darwinismo como por los progresos de la investigación celular, y que tuvo mejores éxitos, fue la establecida por el zoólogo alemán August WeISMann (1885), Die Continuität des Keimplasmas als Grundlage einer Theorie der Vererbung.

52 G. L. L. Conde de Buffon (1707-1788). En el fondo el mecanicismo de Buffon contenía también una idea vitalista cristalizada en su idea de «force pénétrante».

53 C. F. Wolff (1734-1794) En sus explicaciones sobre el crecimiento y el desarrollo rechazó las posturas vitalistas, en su favor creía que las fuerzas esenciales involucradas en estos procesos eran fuerzas puramente físicas, entre ellas las de atracción y repulsión. 
solución aceptable a la incertidumbre, una salida en la frontera del conocimiento, o una explicación de lo desconocido e inaccesible al intelecto humano ya que esos límites entre la ciencia y la fe eran cuestiones, decía Darwin, «demasiado profundas como para ser abordadas por la inteligencia humana».

En la aceptación de ese principio vitalista no solo hay razones metafísicas o problemas con las fronteras del conocimiento, también hay una clara postura filosófica respecto al debate del mecanicismo contra el vitalismo y las causas primarias de los fenómenos biológicos. La postura de Darwin respecto a este debate lo podemos ver con mayor claridad en la diferenciación de sus ideas de la pangénesis y las de Buffon.

Darwin señala que las moléculas orgánicas de Buffon parecen a primera vista ser las mismas que las gémulas de su hipótesis, pero ellas dice son esencialmente diferentes ${ }^{54}$.

Buffon consideraba que un individuo no era más que un todo uniformemente organizado en todas sus partes internas, un compuesto de una infinidad de figuras semejantes y de partes similares o moléculas orgánicas microscópicas, un conjunto de gérmenes y de pequeños individuos de la misma especie, todos los cuales podían desarrollar la misma forma, según las circunstancias, y formaban nuevos seres completos como el primero. Los cuerpos de los vegetales y animales se forman con estas moléculas. Para explicar la capacidad de estas moléculas en la formación de partes y órganos de los seres vivos Buffon supuso la existencia de ciertos moldes internos en los cuerpos y que estos procesos de formación se realizaban bajo la influencia de cierta «fuerza penetrante» [force pénétrante] que actuaba en todos los cuerpos orgánicos. Buffon aventuró la analogía entre esta fuerza y la de la gravedad, de la misma manera como la fuerza de la gravedad penetra en el interior de toda materia, también la fuerza que elabora o atrae las partes orgánicas del alimento se introduce en el interior de los cuerpos orgánicos para llevarlos a ellos por su acción ${ }^{55}$.

Estas ideas de Buffon ya habían sido analizadas y discutidas por el abuelo de Darwin (Erasmus Darwin) en su capítulo de la «Generación» de Zoonomía (1794), reflexiones y discusiones que el nieto releyó en $1839^{56}$, en la misma época que leyó a Blumenbach y a Spallanzani.

54 DARWIN cita La Historia Natural de Buffon de 1749, Tomo 2, pp. 54- 62, 329, 33, 420 y 424, en DARWIN (1875), p. 370.

55 BufFON (1749), Histoire naturelle, Tomo 2, p. 46.

56 En sus primeros escritos Darwin anotó la idea de que la madre construye la «yema» (capullo) y posteriormente ya madura puede recibir la porción vital (semen), no resultaría extraño que la hubiera tomado de E. Darwin. Quien también pensaba en términos similares. 
E. Darwin sostenía que cada parte separada de todo el organismo se reproducía a sí misma y originaba elementos o entidades que serían los responsables del origen de un nuevo ser; en su reflexión negó la versión descrita por Buffon $^{57}$, particularmente porque el autor francés consideraba a las partículas orgánicas como partes mecánicas elaboradas a partir de los fluidos corporales.

E. Darwin no compartió el supuesto materialismo que Buffon expone en sus ideas, porque contradecía su postura claramente vitalista bajo la cual consideraba que «el gran creador de todas las cosas había diversificado infinitamente el trabajo de sus manos» ${ }^{58}$, E. Darwin pensaba que no solo existían en la naturaleza los principios de la gravitación (en el que se basan las concepciones de Buffon) sino también el principio de las afinidades químicas interpretado por E. Darwin como el principio de la vida orgánica, ambos llamados por él, como materia y espíritu, uno y otro considerados como causa de la existencia del mundo natural ${ }^{59}$.

Siguiendo la tradición familiar Ch. Darwin también rechazará en su momento esa postura mecanicista, utilizando el supuesto vitalista del nisus formativus de Blumenbach.

\section{CONCLUSIÓN}

En la segunda edición de The Variation Darwin incorporó una nota señalando la observación de G. G. Lewes ${ }^{60}$ sobre los autores que habían propuesto con anterioridad ideas similares a las de la pangénesis. En esa nota Darwin señala que las ideas de Buffon son muy parecidas a las suyas, pero también señala que hay una diferencia fundamental entre ellas.

\footnotetext{
Tiempo después Darwin extendió la idea del poder vital a todos los elementos sexuales. DARWIN, Ch, Notebook D., p. 388. Respecto a esta idea de fecundación en Darwin John Farley ha hecho una interpretación similar. FARLEY, J. (1982), Gametes and Spores, Ideas About Sexual Reproduction 1750-1914, pp. 107-109.

57 E. Darwin también plantea en sus reflexiones algunas objeciones a la idea pangenética: «primero, porque en las plantas puedan verse ciertos fenómenos que no son aplicables a las leyes animales. Segundo, esos fluidos repletos con partículas orgánicas derivadas tanto de los órganos masculino y femenino, son supuestamente similares y no hay razones por las que una madre no pueda producir un embrión hembra (como ocurre en la partenogénesis) sin la asistencia del macho». DARWIN, E. (1794), Zoonomia: or the Law of Organic Life, p. 530.

58 DARWIN, E. (1794), p. 1.

59 Ibidem, p. 530.

60 DARWIN, Ch. (1875), p. 370.
} 
Como señalamos anteriormente, la diferencia esencial está relacionada con la participación o no de las fuerzas físico-químicas como responsables de los procesos orgánicos. Mientras Buffon basaba sus ideas en un principio análogo a la fuerza gravitatoria como parte de los procesos de la generación, Darwin consideraba necesario un impulso vital o un principio formativo.

Ese principio fue el nisus formativus de Blumenbach, una conjetura postulada precisamente para rechazar ideas mecanicistas de John T. Needham y Wolff. Darwin motivado por las limitaciones del conocimiento de su época, a pesar de la solidez en ese momento de la teoría celular; o por la constante y permanente persistencia de su viejo programa de investigación: la generación; o por sus propias convicciones deístas y filosóficas asumió con ligeras diferencias la concepción vitalista de Blumenbach, sosteniendo la creencia en una fuerza o poder, un tanto metafísico, exclusivo e inherente a toda la organización de los seres vivos.

\section{BIBLIOGRAFÍA:}

BlumenBACH, J. F. (1829), The Elements of Physiology, 4ta. edición, London, Logman.

Bowler, P. (1989), The Mendelian Revolution: The Emergence of Hereditarian Concepts in Modern Science and Society, Johns Hopkins University, Baltimore.

Bowler, P. (1989), Evolution: The History of an Idea, University of California, Berkeley.

Buffon, G. L. L. (1749), Histoire Naturelle, Générale et particulière, Tomo II, Avec la description Du Cabinet du Roy, A Paris, De L'imprimerie Royal.

Canguilhem, G. (1976), El Conocimiento de la Vida, Editorial Anagrama.

DARWIN, Ch. (1868), The Variation of Animals and Plants Under Domestication, Vol. 1 -2, London Murray.

DARWIN, Ch. (1875), The Variation of Animals and Plants Under Domestication, Vol, 1 y 2, Reedición de la 2da edición [1998], The Johns Hopkins University Press.

DARWIN, Ch. (1987), Charles Darwin's Notebooks, 1836-1844: Geology, Transmutation of Species, Metaphysical Enquiries, Paul H. Barrett (ed.), Cornell University Press.

DARWIN, E. (1794), Zoonomia: or the Law of Organic Life, 4ta. edición Americana, Vol. I, Published By Edward Earle.

DE VRIES, H. (1910), Pangenesis Intracellular, The Open Court Publishing, Chicago. 
Delpino, F. (1869), «On the Darwinian Theory of Pangenesis», Scientific Opinion, Sep 29, p. 365-367; Oct 6, pp. 391-393, Oct 13, pp. 407-408.

Di Gregorio, M. A. (1990), Charles Darwin's Marginalia, Vol, 1, Garland Publishing.

FARLEY, J. (1982), Gametes and Spores, Ideas About Sexual Reproduction 17501914, The Johns Hopkins University Press.

Galton, F. (1971), «Experiments in Pangenesis, by Breeding from Rabbits of a Pure Variety, into Whose Circulation Blood Taken from other Varieties had Previously Been Largely Transfused», Proc. Roy. Soc. 19: 393-410.

JACOB, F. (1993), The Logic of the Life: A History of the Heredity, Princeton University Press.

Hodge, M. J. S. (1985), «Darwin as a Lifelong Generation Theorist», en Kohn, D. (ed.), The Darwinian Heritage, Princeton University Press, pp. 207-244.

HodGe, M. J. S. (1989), «Generation and the Origin of the Species (1837-1937): A Historiographical Suggestion», BJHS, pp. 267-281.

López B., C. (1992), Human Heredity 1750 - 1870; The Construction of a Domain, Tesis Doctoral, University of London.

LESKY, E. (1951), «Die Zeuguns- und Vererbungslehren der Antike und ihr Nachwirten» Akad. Wis. Lit. Mainz, Abh. geistes-u. sozialwiss. Kl., Jg. 1950, nr. 19, Wiesbaden, pp. 1227-1425.

MAYR, E. (1982), Growth of Biological Thought, The Belknap Press of Harvard.

NAEGELI, C. (1884), Mechanischphysiologische Theorie der Abstammungslehre, Munich y Leipzig.

Noguera Solano, R. y Ruiz GutiÉRrez, R. «Darwin and Inheritance Biological Problem» (en prensa).

OlBY, R. C. (1963), Origins of Mendelism, Constable, London.

OlBY, R. C. (1963), «Charles Darwin's Manuscript of Pangenesis», The British Journal for the History of Science, Vol. 1, No. 3, pp.250-263.

RADL, E. (1930), The History of Biological Theories, Oxford University, London.

Singer, Ch. (1947), Historia de la Biología, Espasa-Calpe, Argentina.

RichARDS, R. J. (1992), The Meaning of Evolution: The Morphological Construction and the Ideological Reconstruction of Darwin's Theory, The University of Chicago Press.

SlOAN, Ph. R. (1985), «Darwin's Invertebrate Program, 1826-1836 Preconditions forTransformism» en Kohn, D. (ed.), The Darwinian Heritage, Princeton University Press, pp. 71-120. 
Stauffer, R. C. (1987), Natural Selection, Being the Second Part of his Big Species Book Written from 1856 to 1858, Cambridge University Press.

VORZIMMER, P. J. (1977), «The Darwin Reading Notebooks (1836-1860)», Journal of the History of Biology, Vol. 10, no. 1, pp 107-153.

Weismann, A. (1885), Die Continuität des Keimplasmas als Grundlage einer Theorie der Vererbung, Jena.

WiNTHER, R. G. (2000), «Darwin on Variation and Heredity», Journal of the History of Biology, 33: 425-455.

ZIRKLE, C. (1946), «The Early History of the Inheritance of Acquired Characters and Pangenesis», Transactions of the American Philosophical Society, Vol. 35, parte 2, pp.91-150. 